\title{
SUPPLEMENT TO “LIKELIHOOD REWEIGHTING METHODS TO REDUCE POTENTIAL BIAS IN NONINFERIORITY TRIALS WHICH RELY ON HISTORICAL DATA TO MAKE INFERENCE"1:
}

\author{
By Lei Nie ${ }^{1}$, Zhiwei Zhang ${ }^{2 *}$, Daniel Rubin ${ }^{1}$, Jianxiong $\mathrm{Chu}^{2}$ \\ ${ }^{1}$ Division of Biometrics IV, Office of Biostatistics/CDER/FDA \\ 10903 New Hampshire Avenue, Silver Spring, MD 20993 \\ ${ }^{2}$ Division of Biostatistics, Office of Surveillance and Biometrics/CDRH/FDA, \\ 10903 New Hampshire Avenue, Silver Spring, MD 20993
}

In Section 1 of this supplement we provide an assessment of the efficiency loss for the weighted likelihood method. In Section 2 we provide a comparison between the likelihood reweighting method and related methods in historically controlled trials, e.g. Zhang (2007), Signorovitch et al. (2010) and Signorovitch et al. (2011).

Section 1: Tradeoff between accuracy and efficiency

The weighted likelihood method leads to a tradeoff between accuracy and efficiency. Specifically, we will have a gain in terms of a smaller bias, but the gain might be associated with some loss of efficiency. Such a tradeoff exists in many problems that involve bias correction, and is not unique to noninferiority trials or inverse probability weighting. For the scenarios considered in this paper, our experience suggests that the unacceptable efficiency loss only happens when extreme weights occur, caused by factors such as the population heterogeneity and problems in propensity score modeling. If the population heterogeneity is the key factor to the efficiency loss, the problem is intrinsic. We proposed several alternative methods to avoid the large scale of efficiency loss in the

${ }^{1}$ This article reflects the views of the authors and should not be construed to represent FDA's views or policies. 
paper. If problems in propensity score modeling are the key factors, we suggested some general techniques, e.g. GBR, stratification, etc. to ameliorate the problem. We conducted a simulation study to illustrate the scale of and potential reasons for efficiency loss.

Simulation setup 1: In this simulation, we generated a historical trial data with 1200 subjects, randomly receiving treatment $T=1$ or $T=0$. Values of four covariates, $X, X_{1}, X_{2}$, $\mathrm{X}_{3}$ were randomly assigned to 1200 subjects according to a Bernoulli distribution with the event probabilities being $0.3,0.4,0.6,0.5$. We assumed that treatment heterogeneity occurred on $\mathrm{X}$ but that the outcome response variable was not related to $\mathrm{X}_{1}, \mathrm{X}_{2}, \mathrm{X}_{3}$,

$$
y \sim \operatorname{Binomail}(1, p) ; \operatorname{logit}(p)=-1+T+T \times X .
$$

We fit the logistic regression model $\operatorname{logit}(p)=\alpha+\beta T$ to estimate the unadjusted logodds ratio, which applied to the historical population.

We continued to generate a noninferiority trial population with the same structure, but without responsible variable $Y$ nor $T$. Covariates $\mathrm{X}, \mathrm{X}_{1}, \mathrm{X}_{2}, \mathrm{X}_{3}$ were randomly assigned according to the Bernoulli distribution with event probabilities being 0.7, 0.6, 0.5, 0.4 . We fit the propensity score through a logistic regression model, including all covariates ( $\mathrm{X}, \mathrm{X}_{1}, \mathrm{X}_{2}, \mathrm{X}_{3}$ ) or only including $\mathrm{X}$ (the only effect modifier). With this score, we reweighted the likelihood in the historical trial through our method to estimate the logodds ratio.

For the purpose of comparison, we repeatedly generated the historical trial data to mimic redoing a placebo controlled trial in the new noninferiority population. That is, $\mathrm{X}, \mathrm{X}_{1}, \mathrm{X}_{2}$, 
$\mathrm{X}_{3}$ were randomly assigned according to Bernoulli trial with event probabilities being 0.7 , $0.6,0.5,0.4$. We continued to generate the response variable $y \sim \operatorname{Binomail}(1, p)$; $\operatorname{logit}(p)=-1+T+T \times X$.

We call this ideal scenario setup, meaning the historical and noninferiority trials have identical populations. For this historical trial, we estimated the unadjusted log-odds ratio, which applied to the historical population and the noninferiority population. We use this result as a reference. The simulation was repeated 2,000 times and the means of the 2,000 estimates are summarized below.

Table 1: simulation results for setup 1 .

\begin{tabular}{|l|l|l|l|l|}
\hline & $\begin{array}{l}\text { Unadjusted } \\
\text { approach }\end{array}$ & $\begin{array}{l}\text { Weighted } \\
\text { likelihood } \\
\text { approach with all } \\
\text { covariates used } \\
\text { for PS scoring }\end{array}$ & $\begin{array}{l}\text { Weighted } \\
\text { likelihood approach } \\
\text { with only X used } \\
\text { for PS scoring }\end{array}$ & $\begin{array}{l}\text { Ideal Scenario } \\
\text { (reference) }\end{array}$ \\
\hline $\begin{array}{l}\text { Log-odds ratio } \\
\text { (OR) }\end{array}$ & 1.277 & 1.672 & 1.671 & 1.678 \\
\hline $\begin{array}{l}\text { Standard error } \\
\text { of Log OR }\end{array}$ & 0.123 & 0.186 & 0.165 & 0.126 \\
\hline $\begin{array}{l}\text { The lower } \\
\text { bound of the } \\
\text { 95\% C.I. of log } \\
\text { OR }\end{array}$ & 1.035 & 1.308 & 1.348 & 1.430 \\
\hline
\end{tabular}

Simulation setup 2: everything in setup 1 remained the same, except we allowed greater heterogeneity between the historical trial population and the noninferiority trial population by generating $X_{1}, X_{2}, X_{3}$ according to a Bernoulli distribution with event probabilities being $0.8,0.7$, and 0.2 in the noninferiority trial. Note that only the weighted likelihood approach using all covariates for propensity scoring was impacted by this change. In this setup, the mean of log OR, standard error of log OR, and the lower bound of the 95\% C.I. of log OR were 1.678, 0.253 , and 1.182. The standard error of 0.253 was 
remarkably larger than 0.186 in the setup 1 , because the chance of an extreme weight was higher due to the greater population heterogeneity in all three dimensions $X_{1}, X_{2}, X_{3}$. This problem may be solved by excluding $\mathrm{X}_{1}, \mathrm{X}_{2}, \mathrm{X}_{3}$ from propensity score modeling, as we recommended only including potential effect modifiers in propensity scoring. This recommendation is based on our experience and illustrated by this limited study, echoing what was recommended by Cole and Hernan (2008) in a related scenario.

\section{Section 2: some related existing methods in historical controlled trials.}

In addition to the independently proposed use of the weighting in this paper from (Zhang (2007), we note that Signorovitch et al, Signorovitch et al. (2010) and Signorovitch et al. (2011) made an approach to relax the need for patient level data in both the historical trial and the current trial. This sheds lights on one practical limitation of our method, the requirement for patient level data from both trials. As they used one arm from each of two trials, the weight in Signorovitch et al. (2010) and Signorovitch et al. (2011) is the same as in Zhang (2007), which is developed for a single arm historical controlled trial. Their idea is similar to the nonparametric method in Section 4 of Zhang (2007). In the following, we provide a self-assessment of this paper in comparison to Signorovitch et al. (2010) and Signorovitch et al. (2011) and Zhang (2007).

In contrast to non-parametric reweighting of the individual response variable to obtain the mean of response rate as used in Signorovitch et al (2010, 2011) and Zhang (2007), this paper explores a parametric approach that reweights the likelihood function to obtain 
an adjusted treatment effect, which is represented as a parameter in a parametric model. In addition, thee implementation is straightforward using standard software such as SAS, as illustrated through examples in the paper. The parametric approach can be extended easily to model response variables that are subjected to limit of detection (e.g. HIV viral loads do not have exact reading if $<50$ copies/ml) or mixed effect models by simply reweighting the likelihood function from each individual. It may be less simple to extend the previous approaches.

In a framework of single-arm historical controlled trial, the implicit assumption in Zhang (2007) and Signorovitch et al. (2010) and Signorovitch et al. (2011) is that the mean of response variable is constant across two trials. This implies, for example, the response rate of Nilotinib in Signorovitch et al, 2011 (or Palivizumab in our paper) is constant across ENESTnd and DASISION (MPACT and MOTA in our paper) for given covariates. This assumption could be problematic in the noninferiority trials. For example, the event rate of Palivizumab in patient with BPD is 3.9\% in MOTA, which is significantly (Fisher's test p-value $=0.003$ ) smaller than $7.9 \%$, the rate of Palivizumab in patient with BPD in the IMPACT, likely due to the improvement of standard of care from 1996 (IMPACT) to 2004-2006 (MOTA). Thus we may not want to assume that the response rate is constant across trials. Meanwhile, we expect placebo has an improved response rate as well than 1996 due to the same improved standard of care. Therefore, in our paper, when applied to noninferiority trials, we assume constant relative treatment effects across trials, e.g. the odds ratio of Palivizumab and Placebo remains the same in IMPACT and MOTA. 
This method and proposals in Signorovitch et al (2010, 2011) and Zhang (2007) may have one common major limitation of increased variance, which is addressed in this paper but not in theirs (mentioned but not addressed in Signorovitch et al 2010). The weight is obtained through propensity scoring. When the weights of some subjects are too large or too small, as often happens in a setup with small sample size and a large number of covariates, the reweighting methods lead to estimation with large variance, possibly offsetting the reduction in bias under some circumstances. When there are some large or small propensity scores, we propose some possible solutions. One of the solutions is to use stratification method. We also include some more possible solutions, including weight truncation and some recommendations in propensity score modeling to deal with extreme weights.

Finally, we also note a relatively minor point. Although Signorovitch et al. (2010) and Signorovitch et al. (2011) mentioned effective sample size, they did not specify how the standard error of an estimator was computed and how to estimate other metrics such as the odds ratio. This should not be construed as a criticism of their work as they used alternative weighted chi-square testing to test hypothesis rather than to provide point estimates.

\section{Reference}

Cole, S. R., and Hernan, M. A. (2008). Constructing inverse probability weights for marginal structural models. Am J Epidemiol 168, 656-664. 
Signorovitch, J. E., Wu, E. Q., Betts, K. A., et al. (2011). Comparative efficacy of nilotinib and dasatinib in newly diagnosed chronic myeloid leukemia: a matchingadjusted indirect comparison of randomized trials. Curr Med Res Opin 27, 1263-1271. Signorovitch, J. E., Wu, E. Q., Yu, A. P., et al. (2010). Comparative effectiveness without head-to-head trials: a method for matching-adjusted indirect comparisons applied to psoriasis treatment with adalimumab or etanercept. Pharmacoeconomics 28, 935-945. Zhang, Z. (2007). Estimating the current treatment effect with historical control data. JP Journal of Biostatistics 1, 217-247. 